\title{
A High-Accuracy Linear Conservative Difference Scheme for Rosenau-RLW Equation
}

\author{
Jinsong Hu and Yulan Wang \\ School of Mathematics and Computer Engineering, Xihua University, Chengdu 610039, China \\ Correspondence should be addressed to Yulan Wang; wangyulan-math@163.com
}

Received 14 July 2013; Revised 15 September 2013; Accepted 16 September 2013

Academic Editor: Chien-Yu Lu

Copyright (C) 2013 J. Hu and Y. Wang. This is an open access article distributed under the Creative Commons Attribution License, which permits unrestricted use, distribution, and reproduction in any medium, provided the original work is properly cited.

\begin{abstract}
We study the initial-boundary value problem for Rosenau-RLW equation. We propose a three-level linear finite difference scheme, which has the theoretical accuracy of $O\left(\tau^{2}+h^{4}\right)$. The scheme simulates two conservative properties of original problem well. The existence, uniqueness of difference solution, and a priori estimates in infinite norm are obtained. Furthermore, we analyze the convergence and stability of the scheme by energy method. At last, numerical experiments demonstrate the theoretical results.
\end{abstract}

\section{Introduction}

In the study of the dynamics of compact discrete systems, wave-wave and wave-wall interactions cannot be described by the well-known KdV equation. To overcome this shortcoming of $\mathrm{KdV}$ equation, Rosenau proposed the following Rosenau equation in $[1,2]$ :

$$
u_{t}+u_{x x x x t}+u_{x}+u u_{x}=0 \text {. }
$$

Rosenau equation (1) is usually used to describe the dense discrete system and simulate the long-chain transmission model through an L-C flow in radio and computer fields. The existence and uniqueness of solution to (1) were proved by Park in [3]. Rosenau equation is also regarded as the transformation of the Regularized Long Wave (RLW) equation (see [4]):

$$
u_{t}-u_{x x t}+u_{x}+u u_{x}=0
$$

which is usually used to simulate the long wave in nonlinear emanative medium. RLW equation plays important role in the study of nonlinear diffusion wave because it could model lots of physical phenomena. As RLW equation and KdV equation have the same approximative order when they are used to describe motivations, RLW equation could simulate almost all of the applications of KdV equation [5]. Therefore, there are many works about Rosenau equation (1) and RLW equation (2) (see, e.g., [6-23]).

Rosenau-RLW equation is the generalization of Rosenau equation (1) and RLW equation (2). The authors of [24-27] studied the numerical solution of Rosenau-RLW equation. Motivated by the above works, we consider the initialboundary value problem of the following Rosenau-RLW equation:

$$
\begin{gathered}
u_{t}-u_{x x t}+u_{x x x x t}+u_{x}+u u_{x}=0, \\
(x, t) \in\left(x_{L}, x_{R}\right) \times(0, T], \\
u(x, 0)=u_{0}(x), \quad x \in\left[x_{L}, x_{R}\right], \\
u\left(x_{L}, t\right)=u\left(x_{R}, t\right)=0, \quad u_{x x}\left(x_{L}, t\right)=u_{x x}\left(x_{R}, t\right)=0, \\
t \in[0, T],
\end{gathered}
$$

where $u_{0}(x)$ is a smooth function.

As the solitary wave solution of (3) is (see, e.g., [24-26])

$$
u(x, t)=\frac{15}{19} \operatorname{sech}^{4}\left(\frac{\sqrt{13}}{26}\left(x-\frac{169}{133} t\right)\right),
$$

the initial-boundary problem (3)-(5) is in accordance with the Cauchy problem of (3) when $-x_{L} \gg 0, x_{R} \gg 0$. 
It is easy to verify that problem (3)-(5) satisfies the following conservative laws [27]:

$$
\begin{gathered}
Q(t)=\int_{x_{L}}^{x_{R}} u(x, t) d x=\int_{x_{L}}^{x_{R}} u_{0}(x) d x=Q(0), \\
E(t)=\|u\|_{L_{2}}^{2}+\left\|u_{x}\right\|_{L_{2}}^{2}+\left\|u_{x x}\right\|_{L_{2}}^{2}=E(0),
\end{gathered}
$$

where $Q(0)$ and $E(0)$ are both constants only depending on initial data.

$\mathrm{Li}$ and $\mathrm{Vu}-\mathrm{Quoc}$ pointed in [28] that in some areas, the ability to preserve some invariant properties of the original differential equation is a criterion to judge the success of a numerical simulation. It is said in [29] that conservative difference scheme can simulate the conservative law of initial problem well and it could avoid the nonlinear blowup. Therefore, constructing conservative difference scheme is an important and significant job. To our knowledge, the theoretic accuracy of the existing difference scheme for Rosenau-RLW equation (see $[24,25,27])$ is $O\left(\tau^{2}+h^{2}\right)$. Particularly, in [27], the authors proposed a three-level linear conservative difference scheme for problem (3)-(5), whose theoretic accuracy is $O\left(\tau^{2}+h^{2}\right)$. One does not need iteration when solving the equation numerically using this scheme. Henceforth, it could save some computing time. Using Richardson extrapolation idea, we will propose a three-level linear difference scheme which has the theoretic accuracy of $O\left(\tau^{2}+h^{4}\right)$ without refined mesh in this paper. Our scheme simulates the two conservative laws (7) and (8) well. And we will study the a priori estimate, existence, and uniqueness of the difference solution. Furthermore, we shall analyze the convergence and stability.

The rest of this paper is organized as follows. We propose the conservative difference scheme in Section 2 and prove the existence and uniqueness of solution to difference scheme by mathematical induction in Section 3. Section 4 is devoted to the prior estimate, convergence, and stability of the difference scheme. In Section 5, we verify our theoretical analysis by numerical experiments.

\section{Difference Scheme and Its Conservative Law}

Let $h$ and $\tau$ be the uniform stepsize in the spatial and temporal directions, respectively. Denote $h=\left(x_{R}-x_{L}\right) / J, x_{j}=x_{L}+$ $j h(j=-2,-1,0,1, \ldots, J, J+1, J+2)$, and $t_{n}=n \tau(n=$ $0,1,2, \ldots, N$, where $N=[T / \tau])$. Let $u_{j}^{n}$ be the difference approximation of $u(x, t)$ at $\left(x_{j}, t_{n}\right)$; that is, $u_{j}^{n} \approx u\left(x_{j}, t_{n}\right)$. Let

$$
\begin{aligned}
Z_{h}^{0}=\{u & =\left(u_{j}\right) \mid u_{-2}=u_{-1}=u_{0}=u_{J}=u_{J+1} \\
& \left.=u_{J+2}=0, j=-2,-1,0,1, \ldots, J, J+1, J+2\right\} .
\end{aligned}
$$

We define the difference operators, inner product, and norms that will be used in this paper as follows:

$$
\begin{array}{rlrl}
\left(u_{j}^{n}\right)_{x} & =\frac{u_{j+1}^{n}-u_{j}^{n}}{h}, & \left(u_{j}^{n}\right)_{\bar{x}} & =\frac{u_{j}^{n}-u_{j-1}^{n}}{h}, \\
\left(u_{j}^{n}\right)_{\widehat{x}}=\frac{u_{j+1}^{n}-u_{j-1}^{n}}{2 h}, & \left(u_{j}^{n}\right)_{\ddot{x}}=\frac{u_{j+2}^{n}-u_{j-2}^{n}}{4 h},
\end{array}
$$

$$
\begin{gathered}
\left(u_{j}^{n}\right)_{\hat{t}}=\frac{u_{j}^{n+1}-u_{j}^{n}}{2 \tau}, \quad \bar{u}_{j}^{n}=\frac{u_{j}^{n+1}+u_{j}^{n-1}}{2}, \\
\left\langle u^{n}, v^{n}\right\rangle=h \sum_{j=1}^{J-1} u_{j}^{n} v_{j}^{n}, \quad\left\|u^{n}\right\|^{2}=\left\langle u^{n}, u^{n}\right\rangle, \\
\left\|u^{n}\right\|_{\infty}=\max _{1 \leq j \leq J-1}\left|u_{j}^{n}\right| .
\end{gathered}
$$

Lemma 1. It follows from the Cauchy-Schwarz inequality and summation by parts (see [30]) that, for any $u \in Z_{h}^{0}$,

$$
\left\|u_{\ddot{x}}\right\|^{2} \leq\left\|u_{\bar{x}}\right\|^{2} \leq\left\|u_{x}\right\|^{2}, \quad\left\|u_{\bar{x}}\right\|^{2}=\left\|u_{x}\right\|^{2} .
$$

In the paper, $C$ denotes a general positive constant which may have a different value in a different occurrence.

Consider the following finite difference scheme for problem (3)-(5):

$$
\begin{aligned}
& \left(u_{j}^{n}\right)_{\hat{t}}-\frac{4}{3}\left(u_{j}^{n}\right)_{x \bar{x} \hat{t}}+\frac{1}{3}\left(u_{j}^{n}\right)_{\widehat{x} \widehat{x} \hat{t}}+\frac{5}{3}\left(u_{j}^{n}\right)_{x x \overline{x x \hat{t}}} \\
& -\frac{2}{3}\left(u_{j}^{n}\right)_{x \bar{x} \widehat{x} \widehat{x}}+\frac{4}{3}\left(\bar{u}_{j}^{n}\right)_{\widehat{x}}-\frac{1}{3}\left(\bar{u}_{j}^{n}\right)_{\ddot{x}} \\
& +\frac{4}{9}\left[u_{j}^{n}\left(\bar{u}_{j}^{n}\right)_{\widehat{x}}+\left(u_{j}^{n} \bar{u}_{j}^{n}\right)_{\widehat{x}}\right] \\
& -\frac{1}{9}\left[u_{j}^{n}\left(\bar{u}_{j}^{n}\right)_{\ddot{x}}+\left(u_{j}^{n} \bar{u}_{j}^{n}\right)_{\ddot{x}}\right]=0 \text {, } \\
& (j=1,2, \ldots, J-1, n=1,2, \ldots, N-1) \\
& u_{j}^{0}=u_{0}\left(x_{j}\right) \quad(j=1,2, \ldots, J-1), \\
& u_{j}^{1}-\frac{4}{3}\left(u_{j}^{1}\right)_{x \bar{x}}+\frac{1}{3}\left(u_{j}^{1}\right)_{\hat{x} \hat{x}}+\frac{5}{3}\left(u_{j}^{1}\right)_{x x \overline{x x}}-\frac{2}{3}\left(u_{j}^{1}\right)_{x \bar{x} \hat{x} \hat{x}} \\
& =u_{0}\left(x_{j}\right)-\frac{\partial^{2} u_{0}}{\partial x^{2}}\left(x_{j}\right)+\frac{\partial^{4} u_{0}}{\partial x^{4}}\left(x_{j}\right)-\tau \frac{\partial u_{0}}{\partial x}\left(x_{j}\right) \\
& -\tau u_{0}\left(x_{j}\right) \frac{\partial u_{0}}{\partial x}\left(x_{j}\right) \quad(j=1,2, \ldots, J-1), \\
& u^{n} \in Z_{h}^{0},\left(u_{0}^{n}\right)_{x \bar{x}}=\left(u_{J}^{n}\right)_{x \bar{x}}=0, \\
& \left(u_{0}^{n}\right)_{\widehat{x} \widehat{x}}=\left(u_{J}^{n}\right)_{\widehat{x} \widehat{x}}=0 \quad(n=0,1,2, \ldots, N) .
\end{aligned}
$$

The discrete boundary condition (15) is reasonable from the boundary condition of (3)-(5). The following theorem shows how the difference scheme (12)-(15) simulates the conservative law numerically.

Theorem 2. Suppose that $u_{0} \in H_{0}^{2}\left[x_{L}, x_{R}\right]$ and $u(x, t) \in C^{5,3}$. Then the scheme (12)-(15) is conservative for discrete energy; that is,

$$
\begin{gathered}
Q^{n}=Q^{n-1}=\cdots=Q^{0}, \\
E^{n}=E^{n-1}=\cdots=E^{0}
\end{gathered}
$$




$$
\begin{aligned}
& (n=1,2, \ldots, N), \text { where } \\
& Q^{n}=\frac{h}{2} \sum_{j=1}^{J-1}\left(u_{j}^{n+1}+u_{j}^{n}\right)+\frac{2 h}{9} \tau \sum_{j=1}^{J-1} u_{j}^{n}\left(u_{j}^{n+1}\right)_{\widehat{x}} \\
& \quad-\frac{h}{18} \tau \sum_{j=1}^{J-1} u_{j}^{n}\left(u_{j}^{n+1}\right)_{\ddot{x}} \\
& E^{n}=\frac{1}{2}\left(\left\|u^{n+1}\right\|^{2}+\frac{4}{3}\left\|u_{x}^{n+1}\right\|^{2}-\frac{1}{3}\left\|u_{\widehat{x}}^{n+1}\right\|^{2}+\frac{5}{3}\left\|u_{x x}^{n+1}\right\|^{2}\right. \\
& \quad-\frac{2}{3}\left\|u_{x \hat{x}}^{n+1}\right\|^{2}+\left\|u^{n}\right\|^{2}+\frac{4}{3}\left\|u_{x}^{n}\right\|^{2}-\frac{1}{3}\left\|u_{\widehat{x}}^{n}\right\|^{2} \\
& \left.+\frac{5}{3}\left\|u_{x x}^{n}\right\|^{2}-\frac{2}{3}\left\|u_{x \hat{x}}^{n}\right\|^{2}\right) .
\end{aligned}
$$

Proof. Multiplying $h$ in the two sides of (12) and taking summation for $j$, we could obtain from (15) and summation by parts [30] that

$$
\begin{gathered}
h \sum_{j=1}^{J-1} \frac{\left(u_{j}^{n+1}-u_{j}^{n-1}\right)}{2 \tau}+\frac{4}{9} h \sum_{j=1}^{J-1} u_{j}^{n}\left(\bar{u}_{j}^{n}\right)_{\widehat{x}} \\
-\frac{1}{9} h \sum_{j=1}^{J-1} u_{j}^{n}\left(\bar{u}_{j}^{n}\right)_{\ddot{x}}=0 .
\end{gathered}
$$

On the other hand,

$$
\begin{gathered}
h \sum_{j=1}^{J-1} u_{j}^{n}\left(\bar{u}_{j}^{n}\right)_{\widehat{x}}=\frac{h}{2} \sum_{j=1}^{J-1} u_{j}^{n}\left(u_{j}^{n+1}\right)_{\widehat{x}}+\frac{h}{2} \sum_{j=1}^{J-1} u_{j}^{n}\left(u_{j}^{n-1}\right)_{\widehat{x}} \\
=\frac{h}{2} \sum_{j=1}^{J-1} u_{j}^{n}\left(u_{j}^{n+1}\right)_{\widehat{x}}-\frac{h}{2} \sum_{j=1}^{J-1} u_{j}^{n-1}\left(u_{j}^{n}\right)_{\widehat{x}} \\
h \sum_{j=1}^{J-1} u_{j}^{n}\left(\bar{u}_{j}^{n}\right)_{\ddot{x}}=\frac{h}{2} \sum_{j=1}^{J-1} u_{j}^{n}\left(u_{j}^{n+1}\right)_{\ddot{x}}+\frac{h}{2} \sum_{j=1}^{J-1} u_{j}^{n}\left(u_{j}^{n-1}\right)_{\ddot{x}} \\
=\frac{h}{2} \sum_{j=1}^{J-1} u_{j}^{n}\left(u_{j}^{n+1}\right)_{\ddot{x}}-\frac{h}{2} \sum_{j=1}^{J-1} u_{j}^{n-1}\left(u_{j}^{n}\right)_{\ddot{x}}
\end{gathered}
$$

From the definition of $Q^{n}$, we know that (16) could be deduced from (19)-(20).

Taking an inner product of (12) and $2 \bar{u}^{n}$ (i.e., $u^{n+1}+$ $u^{n-1}$ ), we could obtain from boundary condition (15) and summation by parts [30] that

$$
\begin{aligned}
\left\|u^{n}\right\|_{\widehat{t}}^{2}+\frac{4}{3}\left\|u_{x}^{n}\right\|_{\widehat{t}}^{2}-\frac{1}{3}\left\|u_{\widehat{x}}^{n}\right\|_{t}^{2}+\frac{5}{3}\left\|u_{x x}^{n}\right\|_{\widehat{t}}^{2}-\frac{2}{3}\left\|u_{x \hat{x}}^{n}\right\|_{\widehat{t}}^{2} \\
+\frac{8}{3}\left\langle\bar{u}_{\widehat{x}}^{n}, \bar{u}^{n}\right\rangle-\frac{2}{3}\left\langle\bar{u}_{\ddot{x}}^{n}, \bar{u}^{n}\right\rangle+2\left\langle\phi\left(u^{n}, \bar{u}^{n}\right), \bar{u}^{n}\right\rangle \\
-2\left\langle\kappa\left(u^{n}, \bar{u}^{n}\right), \bar{u}^{n}\right\rangle=0,
\end{aligned}
$$

where

$$
\begin{aligned}
& \phi\left(u_{j}^{n}, \bar{u}_{j}^{n}\right)=\frac{4}{9}\left[u_{j}^{n}\left(\bar{u}_{j}^{n}\right)_{\widehat{x}}+\left(u_{j}^{n} \bar{u}_{j}^{n}\right)_{\widehat{x}}\right], \\
& \kappa\left(u_{j}^{n}, \bar{u}_{j}^{n}\right)=\frac{1}{9}\left[u_{j}^{n}\left(\bar{u}_{j}^{n}\right)_{\ddot{x}}+\left(u_{j}^{n} \bar{u}_{j}^{n}\right)_{\ddot{x}}\right] .
\end{aligned}
$$

On the other hand,

$$
\begin{aligned}
& \left\langle\bar{u}_{\widehat{x}}^{n}, \bar{u}^{n}\right\rangle=0, \quad\left\langle\bar{u}_{\ddot{x}}^{n}, \bar{u}^{n}\right\rangle=0, \\
& \left\langle\phi\left(u^{n}, \bar{u}^{n}\right), \bar{u}^{n}\right\rangle \\
& =\frac{4}{9} h \sum_{j=1}^{J-1}\left[u_{j}^{n}\left(\bar{u}_{j}^{n}\right)_{\widehat{x}}+\left(u_{j}^{n} \bar{u}_{j}^{n}\right)_{\widehat{x}}\right] \bar{u}_{j}^{n} \\
& =\frac{4}{9} h \sum_{j=1}^{J-1} u_{j}^{n} \bar{u}_{j}^{n}\left(\bar{u}_{j}^{n}\right)_{\widehat{x}}+\frac{4}{9} h \sum_{j=1}^{J-1}\left(u_{j}^{n} \bar{u}_{j}^{n}\right)_{\widehat{x}} \bar{u}_{j}^{n} \\
& =\frac{4}{9} h \sum_{j=1}^{J-1} u_{j}^{n} \bar{u}_{j}^{n}\left(\bar{u}_{j}^{n}\right)_{\widehat{x}}-\frac{4}{9} h \sum_{j=1}^{J-1} u_{j}^{n} \bar{u}_{j}^{n}\left(\bar{u}_{j}^{n}\right)_{\widehat{x}}=0, \\
& \left\langle\kappa\left(u^{n}, \bar{u}^{n}\right), \bar{u}^{n}\right\rangle \\
& =\frac{1}{9} h \sum_{j=1}^{J-1}\left[u_{j}^{n}\left(\bar{u}_{j}^{n}\right)_{\ddot{x}}+\left(u_{j}^{n} \bar{u}_{j}^{n}\right)_{\ddot{x}}\right] \bar{u}_{j}^{n} \\
& =\frac{1}{9} h \sum_{j=1}^{J-1} u_{j}^{n} \bar{u}_{j}^{n}\left(\bar{u}_{j}^{n}\right)_{\ddot{x}}+\frac{4}{9} h \sum_{j=1}^{J-1}\left(u_{j}^{n} \bar{u}_{j}^{n}\right)_{\ddot{x}} \bar{u}_{j}^{n} \\
& =u_{j}^{n} \bar{u}_{j}^{n}\left(\bar{u}_{j}^{n}\right)_{\ddot{x}}-\frac{1}{9} h \sum_{j=1}^{J-1} u_{j}^{n} \bar{u}_{j}^{n}\left(\bar{u}_{j}^{n}\right)_{\ddot{x}}=0 .
\end{aligned}
$$

Taking (23)-(25) into (21), we have

$$
\begin{aligned}
\frac{1}{2 \tau}\left(\left\|u^{n+1}\right\|^{2}-\left\|u^{n-1}\right\|^{2}\right)+\frac{2}{3 \tau}\left(\left\|u_{x}^{n+1}\right\|^{2}-\left\|u_{x}^{n-1}\right\|^{2}\right) \\
-\frac{1}{6 \tau}\left(\left\|u_{\hat{x}}^{n+1}\right\|^{2}-\left\|u_{\widehat{x}}^{n-1}\right\|^{2}\right)+\frac{5}{6 \tau}\left(\left\|u_{x x}^{n+1}\right\|^{2}-\left\|u_{x x}^{n-1}\right\|^{2}\right) \\
-\frac{1}{3 \tau}\left(\left\|u_{x \hat{x}}^{n+1}\right\|^{2}-\left\|u_{x \hat{x}}^{n-1}\right\|^{2}\right)=0 .
\end{aligned}
$$

From the definition of $E^{n}$, we know that (17) could be obtained by deducing the above equality from 0 to $N-1$.

\section{Solvability}

Theorem 3. The solution of difference scheme (12)-(15) is unique.

Proof. We will use the mathematical induction to prove our theorem. We first note that $u^{0}$ and $u^{1}$ are determined uniquely by (13) and (14).

Suppose that $u^{0}, u^{1}, \ldots, u^{n-1}, u^{n}(n \leq N-1)$ are the unique solution to scheme (12)-(15). Next we prove that there exists unique $u^{n+1}$ which satisfies (12)-(15).

Consider

$$
\begin{gathered}
\frac{1}{2 \tau} u_{j}^{n+1}-\frac{2}{3 \tau}\left(u_{j}^{n+1}\right)_{x \bar{x}}+\frac{1}{6 \tau}\left(u_{j}^{n+1}\right)_{\widehat{x} \widehat{x}}+\frac{5}{6 \tau}\left(u_{j}^{n+1}\right)_{x x \overline{x x}} \\
-\frac{1}{3 \tau}\left(u_{j}^{n+1}\right)_{x \bar{x} \hat{x} \hat{x}}+\frac{2}{3}\left(u_{j}^{n+1}\right)_{\widehat{x}}-\frac{1}{6}\left(u_{j}^{n+1}\right)_{\ddot{x}}
\end{gathered}
$$




$$
\begin{aligned}
& +\frac{2}{9}\left[u_{j}^{n}\left(u_{j}^{n+1}\right)_{\widehat{x}}+\left(u_{j}^{n} u_{j}^{n+1}\right)_{\widehat{x}}\right] \\
& -\frac{1}{18}\left[u_{j}^{n}\left(u_{j}^{n+1}\right)_{\ddot{x}}+\left(u_{j}^{n} u_{j}^{n+1}\right)_{\ddot{x}}\right]=0 \\
& \quad(j=1,2, \ldots, J-1, n=1,2, \ldots, N-1) .
\end{aligned}
$$

Taking an inner product of (27) and $u^{n+1}$, we could obtain from boundary condition (15) and summation by parts [30] that

$$
\begin{aligned}
& \frac{1}{2 \tau}\left\|u^{n+1}\right\|^{2}+\frac{2}{3 \tau}\left\|u_{x}^{n+1}\right\|^{2}-\frac{1}{6 \tau}\left\|u_{\widehat{x}}^{n+1}\right\|^{2}+\frac{5}{6 \tau}\left\|u_{x x}^{n+1}\right\|^{2} \\
& -\frac{1}{3 \tau}\left\|u_{x \widehat{x}}^{n+1}\right\|^{2}+\frac{2}{3}\left\langle u_{\widehat{x}}^{n+1}, u^{n+1}\right\rangle-\frac{1}{6}\left\langle u_{\ddot{x}}^{n+1}, u^{n+1}\right\rangle \\
& +\frac{2 h}{9} \sum_{j=1}^{J-1}\left[u_{j}^{n}\left(u_{j}^{n+1}\right)_{\widehat{x}}+\left(u_{j}^{n} u_{j}^{n+1}\right)_{\widehat{x}}\right] u_{j}^{n+1} \\
& -\frac{h}{18} \sum_{j=1}^{J-1}\left[u_{j}^{n}\left(u_{j}^{n+1}\right)_{\ddot{x}}+\left(u_{j}^{n} u_{j}^{n+1}\right)_{\ddot{x}}\right] u_{j}^{n+1}=0 .
\end{aligned}
$$

Note that

$$
\begin{gathered}
\left\langle u_{\widehat{x}}^{n+1}, u^{n+1}\right\rangle=0, \quad\left\langle u_{\ddot{x}}^{n+1}, u^{n+1}\right\rangle=0, \\
h \sum_{j=1}^{J-1}\left[u_{j}^{n}\left(u_{j}^{n+1}\right)_{\widehat{x}}+\left(u_{j}^{n} u_{j}^{n+1}\right)_{\widehat{x}}\right] u_{j}^{n+1} \\
=h \sum_{j=1}^{J-1} u_{j}^{n} u_{j}^{n+1}\left(u_{j}^{n+1}\right)_{\widehat{x}}+h \sum_{j=1}^{J-1}\left(u_{j}^{n} u_{j}^{n+1}\right)_{\hat{x}} u_{j}^{n+1} \\
=h \sum_{j=1}^{J-1} u_{j}^{n} u_{j}^{n+1}\left(u_{j}^{n+1}\right)_{\widehat{x}}-h \sum_{j=1}^{J-1} u_{j}^{n} u_{j}^{n+1}\left(u_{j}^{n+1}\right)_{\widehat{x}}=0, \\
h \sum_{j=1}^{J-1}\left[u_{j}^{n}\left(u_{j}^{n+1}\right)_{\ddot{x}}+\left(u_{j}^{n} u_{j}^{n+1}\right)_{\ddot{x}} u_{j}^{n+1}\right. \\
=h \sum_{j=1}^{J-1} u_{j}^{n} u_{j}^{n+1}\left(u_{j}^{n+1}\right)_{\ddot{x}}+h \sum_{j=1}^{J-1}\left(u_{j}^{n} u_{j}^{n+1}\right)_{\ddot{x}} u_{j}^{n+1} \\
=h \sum_{j=1}^{J-1} u_{j}^{n} u_{j}^{n+1}\left(u_{j}^{n+1}\right)_{\ddot{x}}-h \sum_{j=1}^{J-1} u_{j}^{n} u_{j}^{n+1}\left(u_{j}^{n+1}\right)_{\ddot{x}}=0 .
\end{gathered}
$$

Furthermore, from Lemma 1, one can easily obtain that

$$
\left\|u_{\hat{x}}\right\|^{2} \leq\left\|u_{x}\right\|^{2}, \quad\left\|u_{x \hat{x}}\right\|^{2} \leq\left\|u_{x x}\right\|^{2} .
$$

Henceforth we could have

$$
\left\|u^{n+1}\right\|^{2}+\left\|u_{x}^{n+1}\right\|^{2}+\left\|u_{x x}^{n+1}\right\|^{2}=0
$$

from (28)-(30).

That is, (27) only admits zero solution. So, there exits unique $u_{j}^{n+1}$ that satisfies (12).

\section{Convergence and Stability of the Difference Scheme}

Suppose that $v(x, t)$ is the solution to problem (3)-(5). Let $v_{j}^{n}=v\left(x_{j}, t_{n}\right)$. Then the truncation error of the difference scheme (12)-(15) is

$$
\begin{gathered}
r_{j}^{n}=\left(v_{j}^{n}\right)_{\hat{t}}-\frac{4}{3}\left(v_{j}^{n}\right)_{x \bar{x} \hat{t}}+\frac{1}{3}\left(v_{j}^{n}\right)_{\hat{x} \hat{x} \hat{t}}+\frac{5}{3}\left(v_{j}^{n}\right)_{x x \bar{x} \bar{x} \hat{t}} \\
-\frac{2}{3}\left(v_{j}^{n}\right)_{x \bar{x} \hat{x} \hat{x} \hat{t}}+\frac{4}{3}\left(\bar{v}_{j}^{n}\right)_{\widehat{x}}-\frac{1}{3}\left(\bar{v}_{j}^{n}\right)_{\ddot{x}} \\
+\varphi\left(v_{j}^{n}, \bar{v}_{j}^{n}\right)-\kappa\left(v_{j}^{n}, \bar{v}_{j}^{n}\right) \\
(j=1,2, \ldots, J-1, n=1,2, \ldots, N-1), \\
v_{j}^{1}-\frac{4}{3}\left(v_{j}^{1}\right)_{x \bar{x}}+\frac{1}{3}\left(v_{j}^{1}\right)_{\widehat{x} \hat{x}}+\frac{5}{3}\left(v_{j}^{1}\right)_{x x \overline{x x}}-\frac{2}{3}\left(v_{j}^{1}\right)_{x \bar{x} \widehat{x} \hat{x}} \\
=u_{0}\left(x_{j}\right)-\frac{\partial^{2} u_{0}}{\partial x^{2}}\left(x_{j}\right)+\frac{\partial^{4} u_{0}}{\partial x^{4}}\left(x_{j}\right)-\tau \frac{\partial u_{0}}{\partial x}\left(x_{j}\right) \\
-\tau u_{0}\left(x_{j}\right) \frac{\partial u_{0}}{\partial x}\left(x_{j}\right)+r_{j}^{0} \quad(n=0,1,2, \ldots, N) . \\
v^{n} \in Z_{h}^{0}, \quad(j=1,2, \ldots, J-1), \\
\left(v_{0}^{n}\right)_{x \bar{x}}=\left(v_{J}^{n}\right)_{x \bar{x}}=0, \quad\left(v_{0}^{n}\right)_{\widehat{x} \hat{x}}=\left(v_{J}^{n}\right)_{\widehat{x} \hat{x}}=0
\end{gathered}
$$

Making use of the Taylor expansion theorem, we know that $\left|r_{j}^{n}\right|=O\left(\tau^{2}+h^{4}\right)$ as $h \rightarrow 0, \tau \rightarrow 0$.

Lemma 4. Assume that $u_{0} \in H_{0}^{2}\left[x_{L}, x_{R}\right], u(x, t) \in C^{5,3}$. Then the solution to problem (3)-(5) satisfies

$$
\begin{gathered}
\|u\|_{L_{2}} \leq C, \quad\left\|u_{x}\right\|_{L_{2}} \leq C, \quad\left\|u_{x x}\right\|_{L_{2}} \leq C, \\
\|u\|_{L_{\infty}} \leq C, \quad\left\|u_{x}\right\|_{L_{\infty}} \leq C .
\end{gathered}
$$

Proof. From (8), we know that

$$
\|u\|_{L_{2}} \leq C, \quad\left\|u_{x}\right\|_{L_{2}} \leq C, \quad\left\|u_{x x}\right\|_{L_{2}} \leq C .
$$

Then

$$
\|u\|_{L_{\infty}} \leq C, \quad\left\|u_{x}\right\|_{L_{\infty}} \leq C,
$$

from the Sobolev inequality.

Theorem 5. Suppose that $u_{0} \in H^{2}\left[x_{L}, x_{R}\right], u(x, t) \in C^{5,3}$. Then the solution to difference scheme (12)-(15) satisfies

$$
\left\|u^{n}\right\| \leq C, \quad\left\|u_{x}^{n}\right\| \leq C, \quad\left\|u_{x x}^{n}\right\| \leq C ;
$$

thus,

$$
\left\|u^{n}\right\|_{\infty} \leq C, \quad\left\|u_{x}^{n}\right\|_{\infty} \leq C \quad(n=1,2, \ldots, N) .
$$


Proof. From Theorem 2 and (30) we can get

$$
\left\|u^{n}\right\|^{2}+\left\|u_{x}^{n}\right\|^{2}+\left\|u_{x x}^{n}\right\|^{2} \leq E^{n}=E^{0}=C ;
$$

that is,

$$
\left\|u^{n}\right\| \leq C, \quad\left\|u_{x}^{n}\right\| \leq C, \quad\left\|u_{x x}^{n}\right\| \leq C .
$$

Then the discrete Sobolev inequality (see [30]) shows that

$$
\left\|u^{n}\right\|_{\infty} \leq C, \quad\left\|u_{x}^{n}\right\|_{\infty} \leq C .
$$

Theorem 6. Suppose that $u_{0} \in H^{2}\left[x_{L}, x_{R}\right], u(x, t) \in C^{5,3}$ Then the solution to difference scheme (12)-(15) $u^{n}$ converges to the solution of problem (3)-(5) in the sense of norm $\|\cdot\|_{\infty}$, and the convergent rate is $\mathrm{O}\left(\tau^{2}+h^{4}\right)$.

Proof. Subtracting (12)-(15) from (32) and letting $e_{j}^{n}=v_{j}^{n}-u_{j}^{n}$, we have

$$
\begin{gathered}
r_{j}^{n}=\left(e_{j}^{n}\right)_{\widehat{t}}-\frac{4}{3}\left(e_{j}^{n}\right)_{x \bar{x} \hat{t}}+\frac{1}{3}\left(e_{j}^{n}\right)_{\widehat{x} \widehat{x} \hat{t}}+\frac{5}{3}\left(e_{j}^{n}\right)_{x x \overline{x x \hat{t}}} \\
-\frac{2}{3}\left(e_{j}^{n}\right)_{x \bar{x} \widehat{x} \widehat{x}}+\frac{4}{3}\left(\bar{e}_{j}^{n}\right)_{\widehat{x}}-\frac{1}{3}\left(\bar{e}_{j}^{n}\right)_{\ddot{x}}+\varphi\left(v_{j}^{n}, \bar{v}_{j}^{n}\right) \\
-\varphi\left(u_{j}^{n}, \bar{u}_{j}^{n}\right)-\kappa\left(v_{j}^{n}, \bar{v}_{j}^{n}\right)+\kappa\left(u_{j}^{n}, \bar{u}_{j}^{n}\right) \\
(j=1,2, \ldots, J-1, n=1,2, \ldots, N-1), \\
r_{j}^{0}=e_{j}^{1}-\frac{4}{3}\left(e_{j}^{1}\right)_{x \bar{x}}+\frac{1}{3}\left(e_{j}^{1}\right)_{\widehat{x} \widehat{x}}+\frac{5}{3}\left(e_{j}^{1}\right)_{x x \overline{x x}} \\
-\frac{2}{3}\left(e_{j}^{1}\right)_{x \bar{x} \widehat{x} \hat{x}} \quad(j=1,2, \ldots, J-1), \\
e^{n} \in Z_{h}^{0}, \quad\left(e_{0}^{n}\right)_{x \bar{x}}=\left(e_{J}^{n}\right)_{x \bar{x}}=0, \\
\left(e_{0}^{n}\right)_{\widehat{x} \hat{x}}=\left(e_{J}^{n}\right)_{\widehat{x} \hat{x}}=0 \quad(n=0,1,2, \ldots, N) .
\end{gathered}
$$

Taking the inner product of (43) and $e^{1}$, and using boundary condition (44), we obtain

$$
\begin{aligned}
\left\|e^{1}\right\|^{2} & +\frac{4}{3}\left\|e_{x}^{1}\right\|^{2}-\frac{1}{3}\left\|e_{\hat{x}}^{1}\right\|^{2}+\frac{5}{3}\left\|e_{x x}^{1}\right\|^{2}-\frac{2}{3}\left\|e_{x \hat{x}}^{1}\right\|^{2} \\
& =\left\langle r^{0}, e^{1}\right\rangle .
\end{aligned}
$$

Noticing that

$$
\left|r_{j}^{0}\right|=O\left(\tau^{2}+h^{4}\right) \quad(j=1,2, \ldots, J-1),
$$

we can obtain that

$$
\left\|e^{1}\right\|^{2}+\left\|e_{x}^{1}\right\|^{2}+\left\|e_{x x}^{1}\right\|^{2} \leq O\left(\tau^{2}+h^{4}\right)^{2},
$$

from the Cauchy-Schwarz inequality and (30), and (44).

Then taking the inner product of (41) and $2 \bar{e}^{n}$, and combining with boundary condition (44) again, one can get

$$
\begin{aligned}
\left\langle r^{n}, 2 \bar{e}^{n}\right\rangle= & \left\|e^{n}\right\|_{\hat{t}}^{2}+\frac{4}{3}\left\|e_{x}^{n}\right\|_{\widehat{t}}^{2}-\frac{1}{3}\left\|e_{\hat{x}}^{n}\right\|_{\hat{t}}^{2}+\frac{5}{3}\left\|e_{x x}^{n}\right\|_{\widehat{t}}^{2} \\
& -\frac{2}{3}\left\|e_{x \hat{x}}^{n}\right\|_{\hat{t}}^{2}+\frac{8}{3}\left\langle\bar{e}_{\widehat{x}}^{n}, \bar{e}^{n}\right\rangle-\frac{2}{3}\left\langle\bar{e}_{\ddot{x}}^{n}, \bar{e}^{n}\right\rangle
\end{aligned}
$$

$$
\begin{aligned}
& +2\left\langle\varphi\left(v^{n}, \bar{v}^{n}\right)-\varphi\left(u^{n}, \bar{u}^{n}\right), \bar{e}^{n}\right\rangle \\
& -2\left\langle\kappa\left(v^{n}, \bar{v}^{n}\right)-\kappa\left(u^{n}, \bar{u}^{n}\right), \bar{e}^{n}\right\rangle .
\end{aligned}
$$

Similar to (23), we have

$$
\left\langle\bar{e}_{\tilde{x}}^{n}, \bar{e}^{n}\right\rangle=0, \quad\left\langle\bar{e}_{\ddot{x}}^{n}, \bar{e}^{n}\right\rangle=0 .
$$

By using Lemma 4, Theorem 5, Lemma 1, and the CauchySchwarz inequality, we can get

$$
\begin{aligned}
& \left\langle\varphi\left(v^{n}, \bar{v}^{n}\right)-\varphi\left(u^{n}, \bar{u}^{n}\right), \bar{e}^{n}\right\rangle \\
& =\frac{4 h}{9} \sum_{j=1}^{J-1}\left[v_{j}^{n}\left(\bar{v}_{j}^{n}\right)_{\widehat{x}}-u_{j}^{n}\left(\bar{u}_{j}^{n}\right)_{\widehat{x}}\right] \bar{e}_{j}^{n} \\
& +\frac{4 h}{9} \sum_{j=1}^{J-1}\left[\left(v_{j}^{n} \bar{v}_{j}^{n}\right)_{\widehat{x}}-\left(u_{j}^{n} \bar{u}_{j}^{n}\right)_{\widehat{x}}\right] \bar{e}_{j}^{n} \\
& =\frac{4 h}{9} \sum_{j=1}^{J-1}\left[v_{j}^{n}\left(\bar{v}_{j}^{n}\right)_{\widehat{x}}-u_{j}^{n}\left(\bar{u}_{j}^{n}\right)_{\widehat{x}}\right] \bar{e}_{j}^{n} \\
& -\frac{4 h}{9} \sum_{j=1}^{J-1}\left(v_{j}^{n} \bar{v}_{j}^{n}-u_{j}^{n} \bar{u}_{j}^{n}\right)\left(\bar{e}_{j}^{n}\right)_{\widehat{x}} \\
& =\frac{4 h}{9} \sum_{j=1}^{J-1}\left[e_{j}^{n}\left(\bar{v}_{j}^{n}\right)_{\widehat{x}}+u_{j}^{n}\left(\bar{e}_{j}^{n}\right)_{\widehat{x}}\right] \bar{e}_{j}^{n} \\
& -\frac{4 h}{9} \sum_{j=1}^{J-1}\left(e_{j}^{n} \bar{v}_{j}^{n}+u_{j}^{n} \bar{e}_{j}^{n}\right)\left(\bar{e}_{j}^{n}\right)_{\widehat{x}} \\
& \leq C\left(\left\|e^{n}\right\|^{2}+\left\|e^{n}\right\|^{2}+\left\|\bar{e}_{\hat{x}}^{n}\right\|^{2}\right) \\
& \leq C\left(\left\|e^{n+1}\right\|^{2}+\left\|e^{n}\right\|^{2}+\left\|e^{n-1}\right\|^{2}\right. \\
& \left.+\left\|e_{x}^{n+1}\right\|^{2}+\left\|e_{x}^{n-1}\right\|^{2}\right), \\
& \left\langle\kappa\left(v^{n}, \bar{v}^{n}\right)-\kappa\left(u^{n}, \bar{u}^{n}\right), \bar{e}^{n}\right\rangle \\
& =\frac{h}{9} \sum_{j=1}^{J-1}\left[v_{j}^{n}\left(\bar{v}_{j}^{n}\right)_{\ddot{x}}-u_{j}^{n}\left(\bar{u}_{j}^{n}\right)_{\ddot{x}}\right] \bar{e}_{j}^{n} \\
& +\frac{h}{9} \sum_{j=1}^{J-1}\left[\left(v_{j}^{n} \bar{v}_{j}^{n}\right)_{\ddot{x}}-\left(u_{j}^{n} \bar{u}_{j}^{n}\right)\right]_{\ddot{x}} \bar{e}_{j}^{n} \\
& =\frac{h}{9} \sum_{j=1}^{J-1}\left[v_{j}^{n}\left(\bar{v}_{j}^{n}\right)_{\ddot{x}}-u_{j}^{n}\left(\bar{u}_{j}^{n}\right)_{\ddot{x}}\right] \bar{e}_{j}^{n} \\
& -\frac{h}{9} \sum_{j=1}^{J-1}\left(v_{j}^{n} \bar{v}_{j}^{n}-u_{j}^{n} \bar{u}_{j}^{n}\right)\left(\bar{e}_{j}^{n}\right)_{\ddot{x}} \\
& =\frac{h}{9} \sum_{j=1}^{J-1}\left[e_{j}^{n}\left(\bar{v}_{j}^{n}\right)_{\ddot{x}}+u_{j}^{n}\left(\bar{e}_{j}^{n}\right)_{\ddot{x}}\right] \bar{e}_{j}^{n}
\end{aligned}
$$


TABLE 1: The errors estimates of numerical solution with various $h$ and $\tau$.

\begin{tabular}{lcccccc}
\hline & \multicolumn{2}{c}{$\tau=0.4, h=0.2$} & \multicolumn{2}{c}{$\tau=0.1, h=0.1$} & \multicolumn{2}{c}{$\tau=0.025, h=0.05$} \\
& $\left\|e^{n}\right\|$ & $\left\|e^{n}\right\|_{\infty}$ & $\left\|e^{n}\right\|$ & $\left\|e^{n}\right\|_{\infty}$ & $\left\|e^{n}\right\|$ & $\left\|e^{n}\right\|_{\infty}$ \\
\hline$t=10$ & $9.496041 e-3$ & $3.527311 e-3$ & $5.870764 e-4$ & $2.186688 e-4$ & $3.679896 e-5$ & $1.371124 e-5$ \\
$t=20$ & $1.558799 e-2$ & $5.383055 e-3$ & $9.659182 e-4$ & $3.320382 e-4$ & $6.028058 e-5$ & $2.073326 e-5$ \\
$t=30$ & $1.901987 e-2$ & $6.232659 e-3$ & $1.176163 e-3$ & $3.836776 e-4$ & $7.326285 e-5$ & $2.389046 e-5$ \\
$t=40$ & $2.083318 e-2$ & $6.558748 e-3$ & $1.286929 e-3$ & $4.032652 e-4$ & $8.004082 e-5$ & $2.503454 e-5$ \\
\hline
\end{tabular}

$$
\begin{array}{r}
-\frac{h}{9} \sum_{j=1}^{J-1}\left(e_{j}^{n} \bar{v}_{j}^{n}+u_{j}^{n} e_{j}^{n}\right)\left(\bar{e}_{j}^{n}\right)_{\ddot{x}} \\
\leq C\left(\left\|e^{n}\right\|^{2}+\left\|\bar{e}^{n}\right\|^{2}+\left\|\bar{e}_{\ddot{x}}^{n}\right\|^{2}\right) \\
\leq C\left(\left\|e^{n+1}\right\|^{2}+\left\|e^{n}\right\|^{2}+\left\|e^{n-1}\right\|^{2}\right. \\
\left.+\left\|e_{x}^{n+1}\right\|^{2}+\left\|e_{x}^{n-1}\right\|^{2}\right), \\
\left\langle r^{n}, 2 e^{n}\right\rangle=\left\langle r^{n}, e^{n+1}+e^{n-1}\right\rangle \\
\leq\left\|r^{n}\right\|^{2}+\left\|e^{n+1}\right\|^{2}+\left\|e^{n-1}\right\|^{2} .
\end{array}
$$

Taking (49)-(50) into (48), we obtain

$$
\begin{gathered}
\left\|e^{n}\right\|_{\hat{t}}^{2}+\frac{4}{3}\left\|e_{x}^{n}\right\|_{\hat{t}}^{2}-\frac{1}{3}\left\|e_{\hat{x}}^{n}\right\|_{\hat{t}}^{2}+\frac{5}{3}\left\|e_{x x}^{n}\right\|_{\hat{t}}^{2}-\frac{2}{3}\left\|e_{x \hat{x}}^{n}\right\|_{\hat{t}}^{2} \\
\leq\left\|r^{n}\right\|^{2}+C\left(\left\|e^{n+1}\right\|^{2}+\left\|e^{n}\right\|^{2}+\left\|e^{n-1}\right\|^{2}\right. \\
\left.+\left\|e_{x}^{n+1}\right\|^{2}+\left\|e_{x}^{n-1}\right\|^{2}\right) .
\end{gathered}
$$

Set

$$
\begin{aligned}
B^{n}= & \left\|e^{n+1}\right\|^{2}+\left\|e^{n}\right\|^{2}+\frac{4}{3}\left\|e_{x}^{n+1}\right\|^{2}+\frac{4}{3}\left\|e_{x}^{n}\right\|^{2} \\
& -\frac{1}{3}\left\|e_{\tilde{x}}^{n+1}\right\|^{2}-\frac{1}{3}\left\|e_{\hat{x}}^{n}\right\|^{2}+\frac{5}{3}\left\|e_{x x}^{n+1}\right\|^{2} \\
& +\frac{5}{3}\left\|e_{x x}^{n}\right\|^{2}-\frac{2}{3}\left\|e_{x \tilde{x}}^{n+1}\right\|^{2}-\frac{2}{3}\left\|e_{x \hat{x}}^{n}\right\|^{2} .
\end{aligned}
$$

Summarizing (51) from 1 to $n$, we get

$$
B^{n} \leq B^{0}+2 \tau \sum_{l=1}^{n}\left\|r^{l}\right\|^{2}+C \tau \sum_{l=0}^{n+1}\left(\left\|e^{l}\right\|^{2}+\left\|e_{x}^{l}\right\|^{2}\right) .
$$

From (42) and (47), we know that

$$
B^{0} \leq O\left(\tau^{2}+h^{4}\right)^{2}
$$

Furthermore,

$$
\tau \sum_{l=1}^{n}\left\|r^{l}\right\|^{2} \leq n \tau \max _{1 \leq l \leq n}\left\|r^{l}\right\|^{2} \leq T \cdot O\left(\tau^{2}+h^{4}\right)^{2} .
$$

Therefore,

$$
\begin{aligned}
\left\|e^{n+1}\right\|^{2} & +\left\|e^{n}\right\|^{2}+\left\|e_{x}^{n+1}\right\|^{2}+\left\|e_{x}^{n}\right\|^{2}+\left\|e_{x x}^{n+1}\right\|^{2}+\left\|e_{x x}^{n}\right\|^{2} \\
\leq & B^{n} \leq O\left(\tau^{2}+h^{4}\right)^{2} \\
& +C \tau \sum_{l=0}^{n+1}\left(\left\|e^{l}\right\|^{2}+\left\|e_{x}^{l}\right\|^{2}+\left\|e_{x x}^{l}\right\|^{2}\right) .
\end{aligned}
$$

The discrete Gronwall inequality (see [30]) implies that

$$
\begin{gathered}
\left\|e^{n}\right\| \leq O\left(\tau^{2}+h^{4}\right), \quad\left\|e_{x}^{n}\right\| \leq O\left(\tau^{2}+h^{4}\right), \\
\left\|e_{x x}^{n}\right\| \leq O\left(\tau^{2}+h^{4}\right) .
\end{gathered}
$$

Then we can obtain

$$
\left\|e^{n}\right\|_{\infty} \leq O\left(\tau^{2}+h^{4}\right)
$$

from the discrete Sobolev inequality.

We could prove the following theorems in a similar way of Theorem 6 .

Theorem 7. Suppose that $u_{0} \in H^{2}\left[x_{L}, x_{R}\right]$ and $u(x, t) \in C^{5,3}$. The solution to difference scheme (12)-(15) $u^{n}$ is stable in the sense of norm $\|\cdot\|_{\infty}$.

\section{Numerical Simulations}

As the difference scheme (12)-(15) is a linear system about $u_{j}^{n+1}$, it does not need iteration when we solve it numerically.

Let $x_{L}=-50, x_{R}=100, T=40$, and

$$
u_{0}(x)=\frac{15}{19} \operatorname{sech}^{4} \frac{x}{2 \sqrt{13}} .
$$

For some different values of $\tau$ and $h$, we list errors at several times in Table 1 and verify the accuracy of the difference scheme in Table 2. The numerical simulation of two conservative quantities (7) and (8) is listed in Table 3.

The stability and convergence of the scheme are verified by these numerical experiments. And it shows that our proposed algorithm is effective and reliable. 
TABLE 2: The numerical verification of theoretical accuracy $O\left(\tau^{2}+h^{4}\right)$.

\begin{tabular}{|c|c|c|c|c|c|c|}
\hline & \multicolumn{3}{|c|}{$\left\|e^{n}(h, \tau)\right\| /\left\|e^{4 n}(h / 2, \tau / 4)\right\|$} & \multicolumn{3}{|c|}{$\left\|e^{n}(h, \tau)\right\|_{\infty} /\left\|e^{4 n}(h / 2, \tau / 4)\right\|_{\infty}$} \\
\hline & $\tau=0.4$ & $\tau=0.1$ & $\tau=0.025$ & $\tau=0.4$ & $\tau=0.1$ & $\tau=0.025$ \\
\hline & $h=0.2$ & $h=0.1$ & $h=0.05$ & $h=0.2$ & $h=0.1$ & $h=0.05$ \\
\hline$t=10$ & - & 16.13800 & 15.95361 & - & 16.13083 & 15.94813 \\
\hline$t=20$ & - & 16.17513 & 16.02370 & - & 16.21215 & 16.01475 \\
\hline$t=30$ & - & 16.17110 & 16.05402 & - & 16.24451 & 16.05986 \\
\hline$t=40$ & - & 16.18828 & 16.07841 & - & 16.26410 & 16.10835 \\
\hline
\end{tabular}

TABLE 3: The numerical simulation for conservative quantities (7) and (8).

\begin{tabular}{|c|c|c|c|c|c|c|}
\hline & \multicolumn{2}{|c|}{$\tau=0.4, h=0.2$} & \multicolumn{2}{|c|}{$\tau=0.1, h=0.1$} & \multicolumn{2}{|c|}{$\tau=0.025, h=0.05$} \\
\hline & $Q^{n}$ & $E^{n}$ & $Q^{n}$ & $E^{n}$ & $Q^{n}$ & $E^{n}$ \\
\hline$t=0$ & 7.59537171309 & 4.26542015423 & 7.59093169500 & 4.26542024995 & 7.59065285879 & 4.26542025595 \\
\hline$t=10$ & 7.59537171275 & 4.26558683637 & 7.59093168588 & 4.26542086750 & 7.59065230880 & 4.26541966402 \\
\hline$t=20$ & 7.59537171886 & 4.26577432049 & 7.59093167701 & 4.26542158265 & 7.59065176013 & 4.26541907347 \\
\hline$t=30$ & 7.59537132913 & 4.26590472629 & 7.59093166975 & 4.26542206996 & 7.59065121035 & 4.26541848035 \\
\hline$t=40$ & 7.59537390280 & 4.26598379748 & 7.59093166785 & 4.26542236189 & 7.59065066159 & 4.26541788802 \\
\hline
\end{tabular}

\section{Acknowledgments}

The authors are very grateful to the anonymous referees for their careful reading and useful suggestions, which greatly improved the presentation of the paper. This work is supported in part by Scientific Research Foundation of Sichuan Provincial Education Department (no. 11ZB009), the Key Scientific Research Foundation of Xihua University (no. Z0912611), and the fund of Key Disciplinary of Computer Software and Theory, Sichuan, China, Grant no. SZD080209-1.

\section{References}

[1] P. Rosenau, "A quasi-continuous description of a nonlinear transmission line," Physica Scripta, vol. 34, pp. 827-829, 1986.

[2] P. Rosenau, "Dynamics of dense discrete systems," Progress of Theoretical Physics, vol. 79, pp. 1028-1042, 1988.

[3] M. A. Park, "On the Rosenau equation," Matemática Aplicada e Computacional, vol. 9, no. 2, pp. 145-152, 1990.

[4] D. H. Peregrine, "Calculations of the development of an unduiar bore," Journal of Fluid Mechanics, vol. 25, pp. 321-330, 1966.

[5] D. J. Kortewag and G. Devries, "On the change of form of long waves advancing in a rectangular canal, and on a new type of long stationary waves," Philosophical Magazine, vol. 39, pp. 422443, 1985.

[6] S. K. Chung and S. N. Ha, "Finite element Galerkin solutions for the Rosenau equation," Applicable Analysis, vol. 54, no. 1-2, pp. 39-56, 1994.

[7] L. Zhang, "A finite difference scheme for generalized regularized long-wave equation," Applied Mathematics and Computation, vol. 168, no. 2, pp. 962-972, 2005.

[8] K. Omrani, F. Abidi, T. Achouri, and N. Khiari, "A new conservative finite difference scheme for the Rosenau equation," Applied Mathematics and Computation, vol. 201, no. 1-2, pp. 3543, 2008.
[9] S. K. Chung, "Finite difference approximate solutions for the Rosenau equation," Applicable Analysis, vol. 69, no. 1-2, pp. 149156, 1998.

[10] S. K. Chung and A. K. Pani, "Numerical methods for the Rosenau equation," Applicable Analysis, vol. 77, no. 3-4, pp. 351369, 2001.

[11] S. A. V. Manickam, A. K. Pani, and S. K. Chung, "A second-order splitting combined with orthogonal cubic spline collocation method for the Rosenau equation," Numerical Methods for Partial Differential Equations, vol. 14, no. 6, pp. 695-716, 1998.

[12] J. Hu and K. Zheng, "Two conservative difference schemes for the generalized Rosenau equation," Boundary Value Problems, vol. 2010, Article ID 543503, 18 pages, 2010.

[13] Y. D. Kim and H. Y. Lee, "The convergence of finite element Galerkin solution for the Roseneau equation," The Korean Journal of Computational \& Applied Mathematics, vol. 5, no. 1, pp. 171-180, 1998.

[14] Q. S. Chang, G. B. Wang, and B. L. Guo, "Conservative scheme for a model of nonlinear dispersive waves and its solitary waves induced by boundary motion," Journal of Computational Physics, vol. 93, no. 2, pp. 360-375, 1991.

[15] D. Bhardwaj and R. Shankar, "A computational method for regularized long wave equation," Computers \& Mathematics with Applications, vol. 40, no. 12, pp. 1397-1404, 2000, Advances in partial differential equations, III.

[16] L. M. Zhang and Q. S. Chang, "A new finite difference method for regularized long wave equation," Chinese Journal on Numerical Methods and Computer Applications, vol. 23, pp. 58-66, 2000.

[17] A. A. Soliman and M. H. Hussien, "Collocation solution for RLW equation with septic spline," Applied Mathematics and Computation, vol. 161, no. 2, pp. 623-636, 2005.

[18] K. R. Raslan, "A computational method for the regularized long wave (RLW) equation," Applied Mathematics and Computation, vol. 167, no. 2, pp. 1101-1118, 2005.

[19] A. Esen and S. Kutluay, "Application of a lumped Galerkin method to the regularized long wave equation," Applied Mathematics and Computation, vol. 174, no. 2, pp. 833-845, 2006. 
[20] J. I. Ramos, "Explicit finite difference methods for the EW and RLW equations," Applied Mathematics and Computation, vol. 179, no. 2, pp. 622-638, 2006.

[21] H. Gu and N. Chen, "Least-squares mixed finite element methods for the RLW equations," Numerical Methods for Partial Differential Equations, vol. 24, no. 3, pp. 749-758, 2008.

[22] Siraj-ul-Islam, S. Haq, and A. Ali, "A meshfree method for the numerical solution of the RLW equation," Journal of Computational and Applied Mathematics, vol. 223, no. 2, pp. 997-1012, 2009.

[23] L. Mei and Y. Chen, "Numerical solutions of RLW equation using Galerkin method with extrapolation techniques," Computer Physics Communications, vol. 183, no. 8, pp. 1609-1616, 2012.

[24] J.-M. Zuo, Y.-M. Zhang, T.-D. Zhang, and F. Chang, "A new conservative difference scheme for the general Rosenau-RLW equation," Boundary Value Problems, vol. 2010, Article ID 516260, 13 pages, 2010.

[25] X. Pan and L. Zhang, "Numerical simulation for general Rosenau-RLW equation: an average linearized conservative scheme," Mathematical Problems in Engineering, vol. 2012, Article ID 517818, 15 pages, 2012.

[26] R. C. Mittal and R. K. Jain, "Numerical solution of general Rosenau-RLW equation using quintic B-splines collocation method," Communications in Numerical Analysis, vol. 2012, Article ID 00129, 16 pages, 2012.

[27] X. Pan and L. Zhang, "On the convergence of a conservative numerical scheme for the usual Rosenau-RLW equation," Applied Mathematical Modelling, vol. 36, no. 8, pp. 3371-3378, 2012.

[28] S. Li and L. Vu-Quoc, "Finite difference calculus invariant structure of a class of algorithms for the nonlinear KleinGordon equation," SIAM Journal on Numerical Analysis, vol. 32, no. 6, pp. 1839-1875, 1995.

[29] Z. Fei, V. M. Pérez-García, and L. Vázquez, "Numerical simulation of nonlinear Schrödinger systems: a new conservative scheme," Applied Mathematics and Computation, vol. 71, no. 23, pp. 165-177, 1995.

[30] Y. L. Zhou, Applications of Discrete Functional Analysis to the Finite Difference Method, International Academic Publishers, Beijing, China, 1990. 


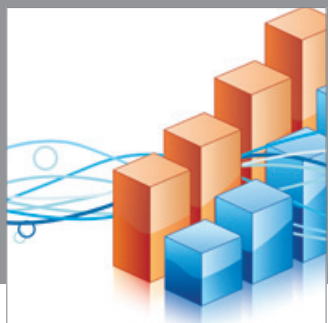

Advances in

Operations Research

mansans

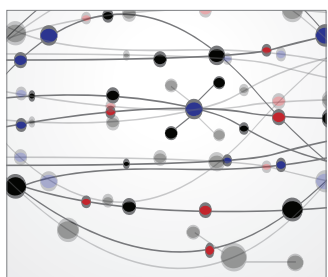

The Scientific World Journal
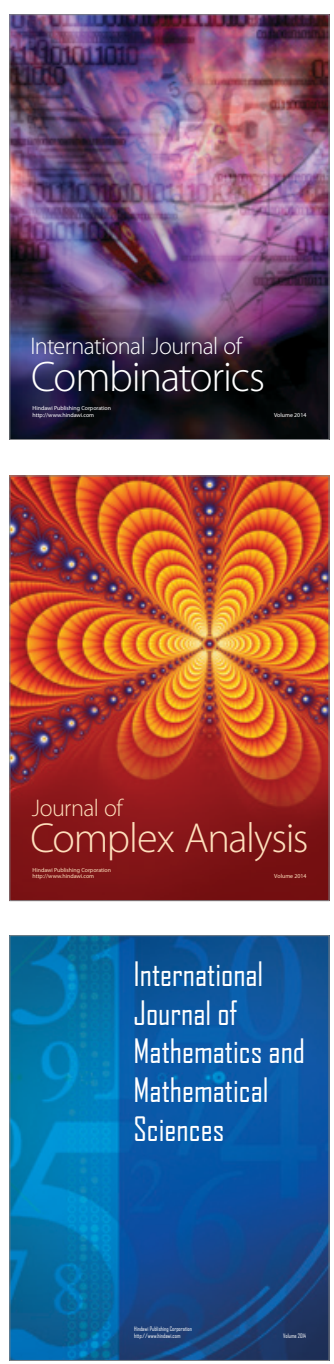
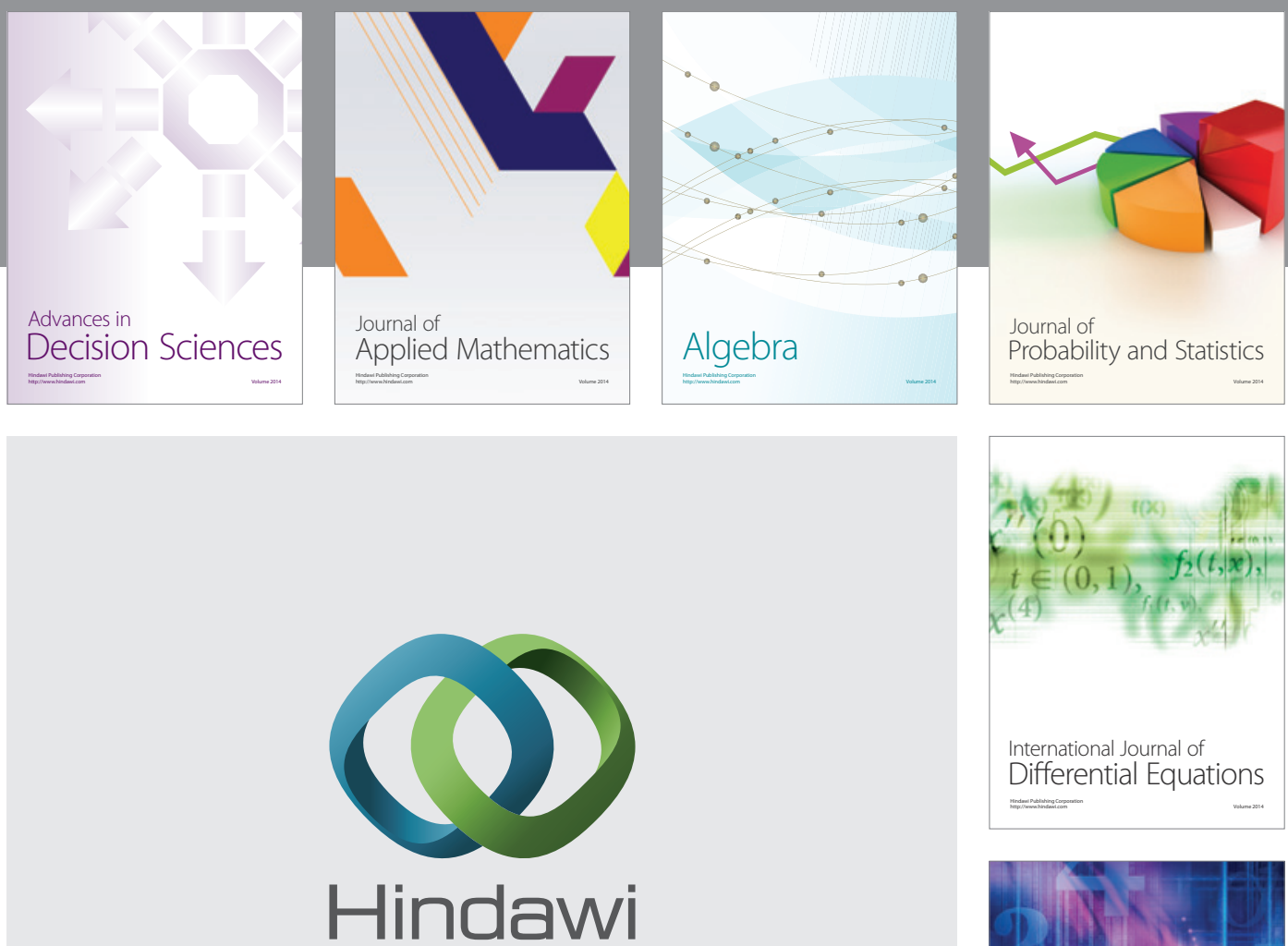

Submit your manuscripts at http://www.hindawi.com
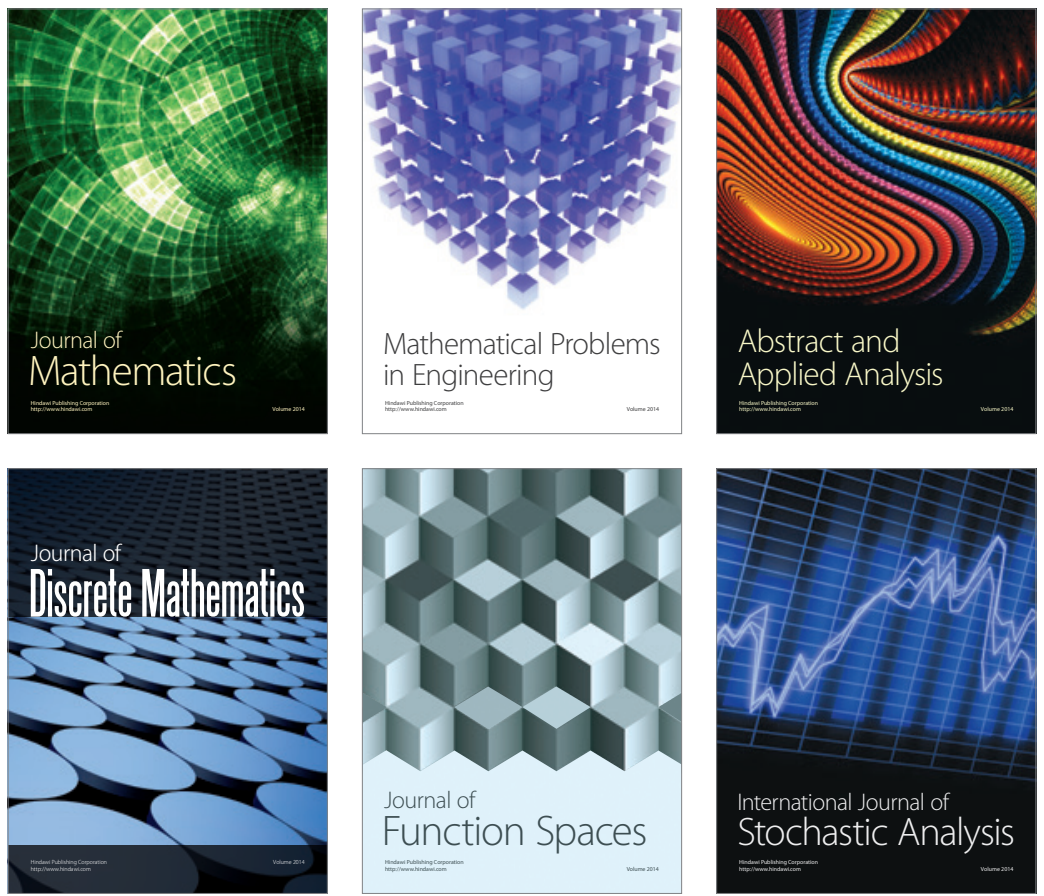

Journal of

Function Spaces

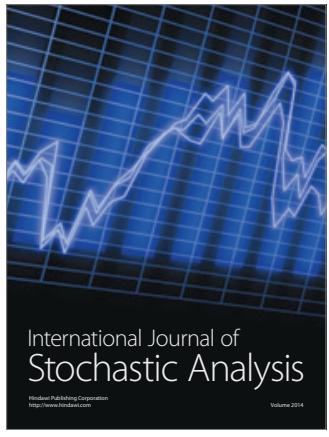

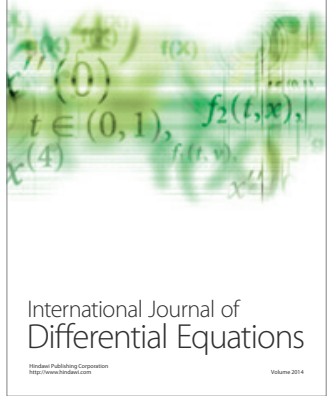
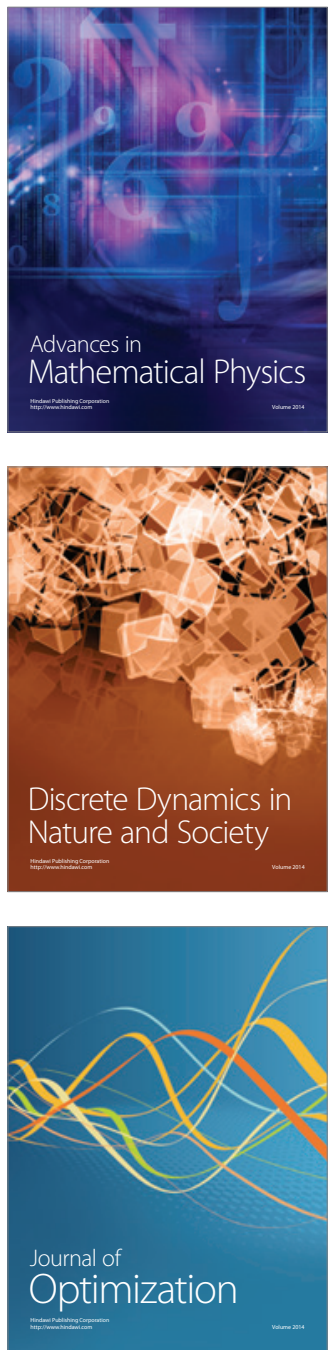